\title{
Die beste aller Welten?
}

Gutachterverfahren sind auch in Wissenschaft und Fachzeitschriften immer wieder Anlass für Lob und Tadel. Bei «offiziellen» Anlässen überwiegt häufig das Lob. Hier wird regelmäßig die Bedeutung des «Peer Review» für die Qualitätskontrolle hervorgehoben. Angesichts mancher dieser offiziellen Verlautbarungen könnte man meinen, wir lebten in der besten aller (wissenschaftlichen) Welten: Lauter objektive, fachkundige Gutachter - selbstverständlich völlig frei von Neid, Eifersüchteleien und Kleinlichkeit - lebten harmonisch mit ebenso perfekten Autoren zusammen. Bei inoffiziellen Zusammenkünften wird dagegen eher Unmut laut. Wer mag schon gerne kritisiert werden, noch dazu anonym, so dass keine Debatte möglich ist und selbst Missverständnisse zähneknirschend hingenommen werden müssen? So gut wie jeder, der sich häufiger dem Peer Review unterworfen hat, kennt Berichte von grotesken, ganz und gar nicht objektiven und manchmal schlichtweg inkompetenten Gutachten.

Längst ist auch das Gutachterverfahren selbst Gegenstand der Forschung geworden und die Ergebnisse sind alles andere als schmeichelhaft. Auch Gutachter sind nicht gefeit vor Besserwisserei, Schlamperei oder Machtspielen. Das Sein kann das Bewusstsein bestimmen, dieser Satz gilt auch für die Gutachterrolle und Macht eröffnet die Möglichkeit zum Machtmissbrauch. Aber wenn es den völlig objektiven «Supergutachter» nicht gibt, wir also nicht in der besten aller (Gutachter-)Welten leben, lohnt sich dann der ganze Aufwand? Gutachter stöhnen übrigens auch über ihre Arbeit, die ja ehrenamtlich erfolgt, meist unter Zeitdruck und häufig ohne adäquate Verstärkung. Und Herausgeber jammern natürlich erst recht, allein schon das Einsammeln der Gutachten ist häufig genug frustrierend. Aber wenn alle klagen, hört sich das nicht eher nach der schlechtesten aller Welten an?

Frei nach Winston Churchill meine ich, dass das System des Peer Review bei richtiger Anwendung eher die am wenigsten schlechte aller bisher bekannten Methoden ist. Wir brauchen Qualität, wir brauchen ein Beurteilungssystem und anonyme Gutachten verbessern die Objektivität des Verfahrens. Um den oben angesprochenen Gefahren zu begegnen, sind vor allem die berühmten «checks and balances» wichtig, in unserem Fall etwa die «Gewaltenteilung» zwischen Gutachtern und Herausgebern. Wichtig ist weiterhin, dass die Rollen von Autoren, Gutachtern und Herausgebern durchlässig sind und dass hier wirklich «Peers», also Gleichgestellte, am Werk sind. Die Amtszeiten für Herausgeber müssen begrenzt sein, Gutachter und Herausgeber müssen auch als Autoren in Erscheinung treten und aus den Autoren von heute müssen die Gut- achter von morgen werden. Wer die Liste unserer Gutachter betrachtet, wird sehen, dass alle auch als Autoren in dieser oder verwandten Zeitschriften aktiv sind.

Vielleicht muss man es nicht extra sagen, vielleicht aber gerade doch: die Beiträge der Herausgeber werden selbstverständlich dem gleichen Begutachtungsprozess unterworfen wie alle anderen auch. Mancher fragt sich dabei, ob nicht die (anonymen) Gutachten seit der Übernahme der Herausgeberrolle strenger geworden sind. Oder gehört das in das Reich der subjektiven Wahrnehmungen? Na ja, wir leben eben nicht in der besten, sondern (vielleicht?) der am wenigsten schlechten aller Wissenschaftswelten. Im Namen der Herausgeber und auch im Interesse der Leser und Autoren der «Verhaltenstherapie» möchte ich zu Beginn dieses Jahres wieder ganz herzlich den Kolleginnen und Kollegen danken, die sich als Gutachter zur Verfügung gestellt haben. Ohne sie wäre die Qualität unser aller Arbeit schlechter.

Jürgen Margraf, Basel

\section{Dank an die Gutachter}

Die Hauptschriftleitung der Zeitschrift VERHALTENSTHERAPIE dankt sehr herzlich allen Gutachtern, die im Jahre 2000 durch ihre Reviews zur Qualität der Zeitschrift in hohem Maße beigetragen haben:

Herr Prof. G. Buchkemper, Tübingen

Herr Prof. G. Dahme, Hamburg

Herr Prof. P. Fiedler, Heidelberg

Herr Dr. S. Fliegel, Münster

Herr Prof. W. Gerber, Kiel

Herr Prof. K. Hahlweg, Braunschweig

Herr Prof. M. Hautzinger, Tübingen

Herr Prof. F. Hohagen, Lübeck

Frau Prof. R. de Jong-Meyer, Münster

Herr Prof. M. Linden, Berlin

Herr Dr. A. Maercker, Dresden

Herr Prof. R. Meermann, Bad Pyrmont

Frau Dr. N. Münchau, Hamburg

Herr Prof. D. Nutzinger, Bad Bramstedt

Herr Dr. H. Peter, Hamburg

Herr Prof. H. Reinecker, Bamberg

Herr Prof. H. Rüddel, Bad Kreuznach

Herr Dr. S. Sulz, München

Herr Prof. D. Vaitl, Gießen

Herr Dr. M. Zaudig, Windach

\section{KARGER \\ (C) 2001 S. Karger GmbH, Freiburg

